\title{
Simultaneous observations of different type images in a scanning electron microscope
}

\author{
A. Yamada, T. Negishi, M. Yamada, Y. Tokiwa, H. Ohashi and H. Kazumori
}

JEOL LTD. 1-2 Musashino 3-chome Akishima Tokyo 196-8558 JAPAN

Image observations with secondary electrons, backscattered electrons and transmission electrons on a scanning electron microscope (SEM) have been conducted separately by using different signal detectors at an optimal condition for each observation. Since the observations have been performed by separate scannings, it has been difficult to obtain those images from exactly the same area and at the same condition due to image drift and contamination. We have developed a system, which makes it possible to simultaneously take the high-resolution images at one time scanning of the primary electron beam. This system provides a very efficient means for various applications of SEM.

FIG.1. shows the block diagram of the simultaneous observation system of the different type images. The four detectors, an upper- and a lower- secondary electron detector, a backscattered electron detector and a transmission electron detector, are attached in a semi-in-lens type scanning electron microscope. The secondary electrons provide information near a specimen surface, backscattered electrons detect the roughness of the surface and compositions, and transmission electrons give information of the compositions. The image memory data are converted from analog to digital, which are compatible with the USB 2.0(Universal Serial Bus) transfer format, and transferred to a PC using a USB line. The USB 2.0 is the standard transmission bus of the present PCs. An image data are transmitted with a $24 \mathrm{MHz}$ clock at a speed of about 12 image sheets with $1280 \times 960$ pixel data per second, which is enough for present live image observation. The present image transfer system can display four images simultaneously on a screen at maximum (FIG.2.). All the images are live images in real time. These images are stored as image memories in a computer as digital data. The stored images can be used for subsequent data processing. The advantages of taking different type images simultaneously are as follows:

1) Different images taken from the same area and at the same observation conditions can be compared. (Each image don't have time delay and position gap.)

2) The image formed by adding the four images can be compared the original images in real time. (The addition ratio of the original images can be adjusted by looking the original images.)

An example of the addition image of the four original images is shown in FIG.3. Various manipulations of the images are possible to find appropriate addition to enhance what one wants to see.

Using this system, we can obtain different image signals simultaneously, which enables us to conduct various data processing. 


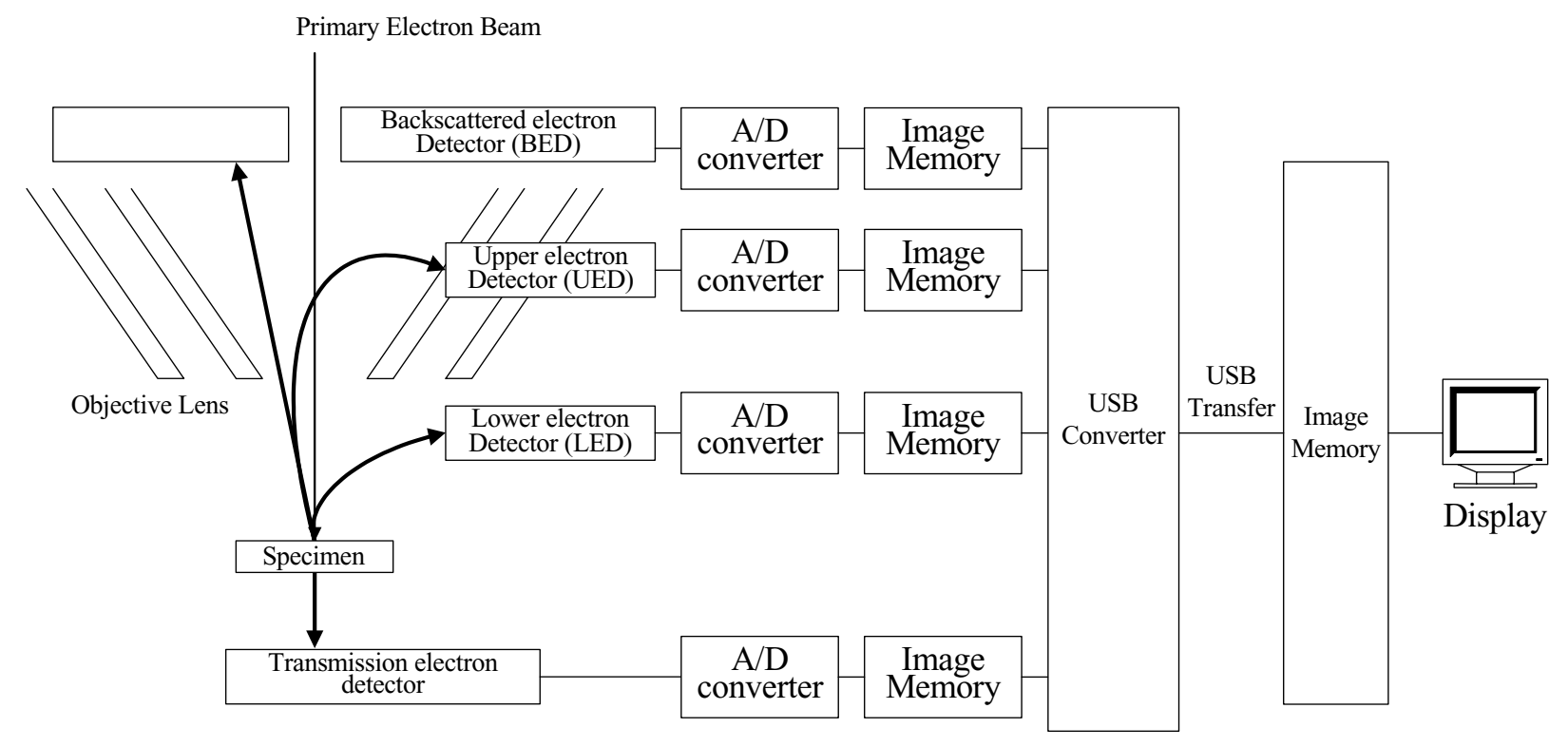

FIG. 1. Block diagram of simultaneous observation system of different type images. The four different images are simultaneously shown on a display in real time.

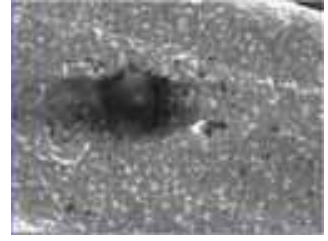

a)UED image

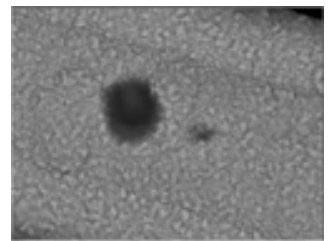

c)BED image(compo.) d)BED image(topo)

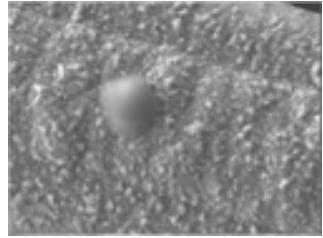

b)LED image

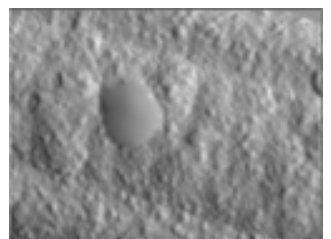

FIG. 2. The four images displayed on a screen as live images, which can be stored as images files in a computer

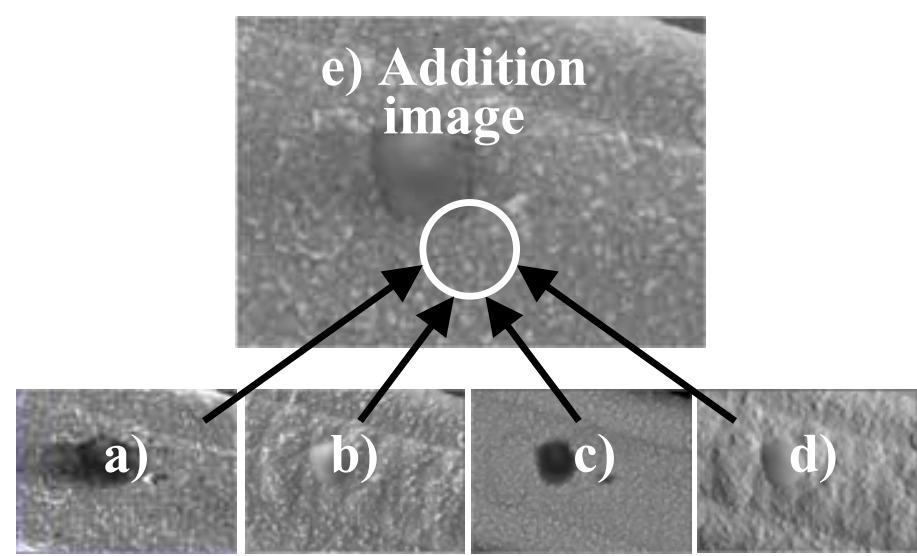

FIG. 3. An example of the addition image e) of the original images: $a), b), c$ ) and d). 